OPEN ACCESS

Edited by:

Sarah Whittle,

University of Melbourne, Australia

Reviewed by:

Carlo Lai

Sapienza University of Rome, Italy Margaret M. Swingler,

University of North Carolina

at Greensboro, USA

${ }^{*}$ Correspondence:

Xu Chen

chenxu@swu.edu.cn

Specialty section:

This article was submitted to Personality and Social Psychology, a section of the journal

Frontiers in Psychology

Received: 06 November 2016 Accepted: 05 April 2017

Published: 21 April 2017

Citation:

Ma Y, Ran G, Chen X, Ma H and Hu N (2017) Adult Attachment Styles

Associated with Brain Activity

in Response to Infant Faces

in Nulliparous Women: An

Event-Related Potentials Study.

Front. Psychol. 8:627.

doi: 10.3389/fpsyg.2017.00627

\section{Adult Attachment Styles Associated with Brain Activity in Response to Infant Faces in Nulliparous Women: An Event-Related Potentials Study}

\author{
Yuanxiao Ma ${ }^{1,2}$, Guangming $\operatorname{Ran}^{3}, \mathrm{Xu}$ Chen ${ }^{1,2 *}$, Haijing $\mathrm{Ma}^{4}$ and $\mathrm{Na} \mathrm{Hu}^{1,2}$ \\ ${ }^{1}$ Faculty of Psychology, Southwest University, Chongqing, China, ${ }^{2}$ Research Center of Mental Health Education, Southwest \\ University, Chongqing, China, ${ }^{3}$ Institute of Education, China West Normal University, Nanchong, China, ${ }^{4}$ School of Foreign \\ Languages for Business, Southwestern University of Finance and Economics, Chengdu, China
}

Adult attachment style is a key for understanding emotion regulation and feelings of security in human interactions as well as for the construction of the caregiving system. The caregiving system is a group of representations about affiliative behaviors, which is guided by the caregiver's sensitivity and empathy, and is mature in young adulthood. Appropriate perception and interpretation of infant emotions is a crucial component of the formation of a secure attachment relationship between infant and caregiver. As attachment styles influence the ways in which people perceive emotional information, we examined how different attachment styles associated with brain response to the perception of infant facial expressions in nulliparous females with secure, anxious, and avoidant attachment styles. The event-related potentials of 65 nulliparous females were assessed during a facial recognition task with joy, neutral, and crying infant faces. The results showed that anxiously attached females exhibited larger N170 amplitudes than those with avoidant attachment in response to all infant faces. Regarding the P300 component, securely attached females showed larger amplitudes to all infant faces in comparison with avoidantly attached females. Moreover, anxiously attached females exhibited greater amplitudes than avoidantly attached females to only crying infant faces. In conclusion, the current results provide evidence that attachment style differences are associated with brain responses to the perception of infant faces. Furthermore, these findings further separate the psychological mechanisms underlying the caregiving behavior of those with anxious and avoidant attachment from secure attachment.

Keywords: attachment style, sensitivity, affective perception, infant facial expression, event-related potentials

\section{INTRODUCTION}

Just like the basic needs for food and water in our evolutionary history, the need to be loved and cared for is deeply rooted in individual psychology, especially in infancy. The parent-infant bond, which is central to the human condition, provides the infant with his/her primary social experience (Swain et al., 2014). The quality of interaction between the infant and caregiver is critical for the infant's survival and development across mammalian species (Swain et al., 2011).

Abbreviations: ANOVA, analysis of variance; ECR, Experiences in Close Relationships inventory; EEG, electroencephalography; ERPs, event-related potentials; RTs, reaction times. 
Indeed, the caregiving system is a subset of maternal behaviors intending to improve closeness and comfortableness when the caregiver detects internal or external cues associated with situations that she perceives as stressful for the child (Lenzi et al., 2013,2015). This suggests that the caregiving system is important for organizing and regulating the infant's emotional, social, and cognitive systems, which is consistent with the accumulating evidence that the quality of caregiving behavior predicts a range of social-emotional child outcomes, including the quality of attachment relationships (Bakermans-Kranenburg et al., 2003; Swain et al., 2014). Moreover, it should be noted that the caregiving system is immature until late adolescence. During puberty, interactions with environmental stimuli, previous attachment experiences, and hormonal and neurobiological changes create a sensitive period that pushes the caregiving system toward maturity (Ammaniti et al., 2000; Grossmann et al., 2006; Lenzi et al., 2013). Through such transformations, late adolescents and young adults, particularly females, show thoughtfulness concerning mothering and they start to present themselves as future parents (George and Solomon, 1999; Lenzi et al., 2013).

The attachment system and caregiving system are complementary systems that are active simultaneously during infant-caregiver interactions (Lenzi et al., 2015). Attachment theory (Bowlby, 1969) postulates that humans are born with a psychobiological system that motivates them to seek proximity to significant others (the mother in particular) in times of need, with the aim of acquiring a feeling of security. Although all infants attach to their caregiver, they manifest different attachment styles depending on the quality of the care they receive. According to Bowlby (1969), the internal working model of attachment is gradually internalized during the interactions between infants and their primary caregiver (usually the mother). When the caregiver is available, responsive, and sensitive and infants are experiencing emotional and physical needs, they tend to manifest patterns of secure attachment. Conversely, chaotic, unpredictable, rejecting, or neglectful care in which non-contingent responses to the child occur frequently lead to an insecure attachment style (Vrtička and Vuilleumier, 2012; Donges et al., 2012). The infant's current and future behavior is significantly shaped during the infant-caregiver interaction. Early childhood appears to be a critical period for the development of the internal working model of attachment (Leyh et al., 2016b). During this developmental period, neural circuits become organized and adjust to the child's environment, which is why these neuronal changes are greatly affected by experiences with the attachment figure during the early years (Luby et al., 2012; Whittle et al., 2015). The parent-infant interaction provides infants with their first social environment and forms templates for what they can expect from others and how best to interact with them (Swain, 2008). In addition, there is good empirical evidence for the transmission of attachment security or insecurity from the parental generation to the child (van Ijzendoorn, 1995; Obegi et al., 2004).

Differences in attachment style may predict a selective bias in attention toward certain types of emotional information in the external environment (Edelstein and Gillath, 2008).
Caregivers who themselves experience a secure attachment are known to have the ability to perceive the infant's signals, to interpret them correctly, and to respond to them appropriately (Grossmann et al., 1985; Fraedrich et al., 2010; Leyh et al., $2016 a, b)$. Indeed, the parent's ability to govern their infant's distress and fear is crucial to the infant's ultimate feeling of security (Lenzi et al., 2013). Anxiously attached individuals have a strong need for closeness, worry about relationships, and fear being rejected (Mikulincer and Shaver, 2007; Vrtička et al., 2012). Consequently, such individuals are thought to hyperactivate the attachment system, become highly sensitive and vigilant to potential threat information and devote more cognitive resources to attachment-related material, threat stimuli in particular (Gillath et al., 2005). In contrast to attachment anxiety, in the case of attachment avoidance, proximity seeking is perceived as futile or even dangerous because of the distress felt by failing to achieve proximity to an attachment figure (Vrtička and Vuilleumier, 2012). An avoidantly attached person is marked by compulsive self-reliance and a preferential use of deactivating strategies to keep psychological distance from other people (Vrtička and Vuilleumier, 2012; Vrtička et al., 2012). Accordingly, avoidantly attached individuals tend to have a restricted ability in terms of perceiving and communicating the infant's feelings and integrating emotional information during parent-infant interaction (Fraedrich et al., 2010). Therefore, the differences in how individuals respond to infants' needs may be based on individual differences in how they maintain social bonds, namely, their attachment styles.

The present study employed a facial recognition task. Infants' facial expressions serve as a key component in conveying information about their emotional states and the specific features of infants' faces contribute to motivate maternal care (Glocker et al., 2009; Leyh et al., 2016a). When investigating the neurophysiological processes of emotional face recognition, different event-related components of the EEG come into play. EEG-ER Ps, a technique used to investigate the temporal brain dynamics of attentive processing at high temporal resolution, was the method employed in current study. The present study focused mainly on the N170 and P300 ERP components. The N170 component is associates with sensitivity to faces and reflects the processing of the configural features of human faces (Eimer and Holmes, 2007; Leyh et al., 2016b). A higher amplitude of the N170 component indicates a higher need for face discrimination resources (Fraedrich et al., 2010; Luo et al., 2016). In addition, top-down control mechanisms operate during the later processing stages and reflect in late ERP responses ( $>300 \mathrm{~ms}$ ), which are interpreted as correlates of attention allocation, arousal, control, and/or awareness (Polich, 2007). For example, as mentioned above, the deactivating strategy of an avoidantly attached group was found to be a product of an effortful strategy that occurred rather late in the information processing sequence (Sonnby-Borgström and Jönsson, 2004). Therefore, the P300 component may be particularly useful as it is thought to be a measure of motivated attention and indicates the amount of "motivational relevance" perceived in a stimulus. For example, increases in the motivational relevance of stimuli are reflected in larger P300 amplitudes (Schupp et al., 2000). 
People's attachment styles are also assumed to affect the development and maturation of the brain and have long-lasting effects on brain structures and brain function (Schore, 1994). A recent study focused on the ERPs of mothers during the perception of infants' emotions by presenting positive, negative, and neutral facial expressions as well as non-facial stimuli within an oddball paradigm (Fraedrich et al., 2010). The study found that insecurely attached mothers demonstrated enhanced N170 amplitudes for the target facial stimuli in the conditions that containing frequent non-facial stimuli. In addition, securely attached mothers showed more pronounced positive P300 amplitudes for target infant faces in the conditions with frequent non-facial stimuli than in the conditions with frequent infant facial stimuli. Furthermore, Leyh et al. (2016a) examined the characteristics of perceptual processing in mothers with different attachment styles by using the oddball paradigm with positive, negative, and neutral infant facial expressions. When the target depicted an infant with negative emotions, insecurely attached mothers exhibited more negative N170 amplitudes than securely attached mothers after the presentation of the target faces with negative emotions, whereas securely attached mothers showed larger P300 amplitudes for the target infant faces than insecurely attached mothers, especially in the conditions with frequent negative infant emotions.

While such recent studies have provided evidence of how attachment-related differences affect the neuropsychological processing of infant facial expressions (Fraedrich et al., 2010; Leyh et al., 2016a), all of the above-mentioned studies have, surprisingly, focused on the difference between securely and insecurely attached groups. However, insecure attachment is more appropriately conceptualized in terms of anxiety and avoidance (Brennan et al., 1998). Differences in anxious and avoidant attachment may predict the perception of, and processing biases toward, certain types of emotional information. Indeed, the behavioral patterns displayed by avoidantly and anxiously attached individuals have been shown to be opposite in almost every respect (Dan and Raz, 2012). Therefore, it is necessary to investigate the neurophysiological processes of infant face recognition in different attachment styles, especially in anxiously and avoidantly attached individuals.

In summary, the purpose of the present study was to test whether participants' brain responses during the processing of infant facial expressions would be affected by their attachment styles. Based on the findings of previous study (Fraedrich et al., 2010; Leyh et al., 2016a), we proposed the following hypotheses. For the N170 component, we expected the N170 amplitudes of anxiously attached individuals to be significantly larger than those of avoidantly attached individuals in response to all infant facial expressions. Since anxiously attached individuals tend to heighten cognitive accessibility to attachment-related material due to their hyperactivity (Mikulincer et al., 2002; Gillath et al., 2005), while avoidantly attached individuals tend to perform avoidance processes and may reflect less and elaborate less on the emotional experiences they have encoded (Vrtička et al., 2008; Lenzi et al., 2013). Indeed, Zhang et al. (2008) found reduced negative ERP components to faces for avoidantly attached subjects compared to anxiously or securely attached subjects. They suggested avoidantly attached individuals are less elaborative in encoding structural information of faces.

As exemplified by larger P300 amplitudes for securely attached individuals in previous studies (Fraedrich et al., 2010; Leyh et al., 2016a), such individuals may associate with more sensitive caregiving behavior, namely the ability to perceive and interpret the infant's signals correctly and timely. The larger P300 amplitudes presented in securely attached individuals may reflect an ability to allocate more attentional resources to infant facial expressions and occurs as a result of experiencing more sensitive caregiving behavior themselves.

Regarding the P300 component, securely attached individuals are associated with more sensitive caregiving behavior and the ability to perceive and interpret the infant's signals correctly, which is exemplified by larger P300 amplitudes for secure attachment, so they can concentrate and allocate more attentional resources to infant facial expressions. Therefore, we assumed that the P300 amplitudes of securely attached individuals would be greater than those of avoidantly attached individuals in response to all infant facial expressions. Moreover, we expected larger P300 amplitudes in anxiously attached individuals than in avoidantly attached individuals in response to crying infant faces because anxiously attached individuals have a strong demand for intimacy, worry about relationships, and fear being rejected, and the crying infant faces may represent a threat to the abovementioned attributes. At the behavioral level, we assumed that securely attached individuals would have the shortest RTs and anxiously attached individuals would have the longest RTs, this is because the former are more sensitive to infants' emotional expressions and needs, while the latter may be immersed in the configural processing of the infant faces.

\section{MATERIALS AND METHODS}

\section{Participants}

We recruited 304 nulliparous female college students to complete the ECR (Brennan et al., 1998) inventory prior to the experiment, which was used to assess the participants' attachment styles. According to screening criteria (Zilber et al., 2007; Chavis and Kisley, 2012; Dan and Raz, 2012), 65 females met the criteria for experiment. Participants were assigned to three attachment groups according to their score on the anxiety and avoidance subscales of the ECR questionnaire. Participants were categorized in experiment as follows: 20 avoidant attachment; 25 anxious attachment; and 20 secure attachment. All participants were healthy, right-handed, and reported normal or corrected-tonormal vision, and none of them had a prior history of neurological or psychiatric disorders.

\section{Ethics Statement}

The ethics committee of the Southwest University of China approved this study and the recruitment of participants. Written informed consent was obtained from all participants prior to conducting the formal experiment, and all participants gave 
written informed consent in accordance with the Declaration of Helsinki. All methods were carried out in accordance with the approved guidelines.

\section{Attachment Scale}

The ECR (Brennan et al., 1998) was designed as a dimensional measure of adult attachment styles, including anxiety and avoidance. According to Zilber et al. (2007), Chavis and Kisley (2012), and Dan and Raz's (2012) criteria, those scoring higher than $1 S D$ above the mean on the anxiety subscale and lower than $1 S D$ below the mean on the avoidance subscale were assigned to the anxiously attached group; those scoring higher than 1 $S D$ above the mean on the avoidance subscale and lower than $1 S D$ below the mean on the anxiety subscale were assigned to the avoidantly attached group; those scoring lower than $1 S D$ below the mean on both the anxiety and avoidance subscales were assigned to the securely attached group. The mean and standard deviations of anxiety and avoidant subscale for each attachment group were presented in Table 1 . The avoidance subscale includes an 18 -item scale $(\alpha=0.94)$ that reflects avoidance of intimacy and interdependence. The anxiety subscale includes an 18 -item scale $(\alpha=0.90)$ that reflects an individual's concern about rejection and abandonment. Each item is rated on a 7 -point Likert scale ranging from 1 (strongly disagree) to 7 (strongly agree). The ECR questionnaire is considered a stable and test-retest reliable (Lopez and Gormley, 2002) measure of an individual's attachment style.

\section{Stimuli and Procedure}

The experimental stimuli consisted of 120 black-and-white photographs of infants aged from 3 to 6 months that were selected from the Chinese Infant Affective Face Picture System (Cheng et al., 2015). The infant facial expressions were either joy, neutral, or crying and 40 pictures were used for each facial expression (50\% female). All infant face pictures were identical in size, brightness, contrast, and spatial frequency. Each infant face was presented twice, generating a total of 240 stimuli that were divided into four blocks of 60 trials each. In the experimental session, infant emotions were displayed with equal probability and their presentation was randomized between trials.

Participants were seated in a quiet room approximately $80 \mathrm{~cm}$ from a computer screen and were required to try their best to avoid eye blinks and head movements. Each trial started with a $500 \mathrm{~ms}$ presentation of a black fixation point followed by a $500 \mathrm{~ms}$ appearance of the display of the infant face (joy, neutral, or crying). The infant face was then replaced by a $300 \mathrm{~ms}$ white

TABLE 1 | ECR scores, and others descriptive statistics of the three attachment groups.

\begin{tabular}{lcccc}
\hline & Total & \multicolumn{1}{c}{$\begin{array}{l}\text { Secure } \\
\text { group }\end{array}$} & $\begin{array}{c}\text { Anxious } \\
\text { group }\end{array}$ & $\begin{array}{c}\text { Avoidant } \\
\text { group }\end{array}$ \\
\hline $\mathrm{N}$ & 65 & 20 female & 25 female & 20 female \\
Age & $21.56 \pm 1.64$ & $21.32 \pm 0.72$ & $21.71 \pm 0.56$ & $21.53 \pm 0.83$ \\
Anxious score & $4.11 \pm 0.53$ & $3.01 \pm 0.13$ & $5.83 \pm 0.18$ & $3.18 \pm 0.13$ \\
Avoidant score & $3.75 \pm 0.69$ & $2.88 \pm 0.11$ & $3.04 \pm 0.14$ & $5.28 \pm 0.16$
\end{tabular}

blank screen. Following the $300 \mathrm{~ms}$ white blank screen display, a black dot was presented on the computer screen. Participants were required to press " 1 " on the response box with their right hand if the target was a joy infant face, " 2 " if it was a neutral infant face, and " 3 " if it was a crying infant face. The dot presentation on the screen was terminated once the participant responded. Following the participant's response, the screen went blank for an inter-trial interval of $1000 \mathrm{~ms}$ with a random jitter of $\pm 200 \mathrm{~ms}$. A schematic illustration of the procedure is shown in Figure $\mathbf{1 .}$ Prior to the experiment, a short practice block was available to familiarize the participants with the experimental procedure.

\section{ERP Recording}

The EEG was recorded, using the BrainAmp system (Brain Products, Munich, Germany), from 64 scalp sites according to the 10-20 system positions with a reference at FCz (Herrmann et al., 2007). A common average reference was recalculated. Vertical electrooculograms were recorded using electrodes placed below the right eye. Horizontal electrooculograms were recorded from the left and right orbital rim. Electrode impedance was maintained at below $5 \mathrm{k} \Omega$. Signals were amplified using a $0.01-100 \mathrm{~Hz}$ band pass filter and continuously sampled at $500 \mathrm{~Hz} /$ channel for off-line analysis.

\section{ERP Analysis}

The ERP data were analyzed off-line using the BrainVision Analyzer (Brain Products, Munich, Germany). The EEG activity was separately averaged for correct responses in each condition. All EEG signals were re-referenced off-line on the left and right mastoids (TP9 and TP10). The EEG data were digitally filtered with a $30 \mathrm{~Hz}$ low-pass filter and were epoched into a period of $1200 \mathrm{~ms}$ (200 ms baseline and $1000 \mathrm{~ms}$ post stimulus onset). The analysis of the electrophysiological data focused on selected electrode sites based on previous findings. The N170 component (140-180 ms) was determined over the P5/6, P7/8, and PO7/8 electrodes (Righart and De Gelder, 2008; Ran et al., 2014). Peak amplitudes and latencies of the N170 component were subjected to a repeated-measures ANOVA with attachment style (secure vs. anxious vs. avoidant) as the betweenparticipants factor, and infant expression (joy vs. neutral vs. crying) and hemisphere (right vs. left) as the within-participants factors. Given the absence of a sharply defined peak, the P300 component (300-500 ms after the target stimuli onset) was observed and quantified for mean amplitudes at CP1/CP2, P1/P2, PO3/4, CPz, Pz, and POz (Tacikowski and Nowicka, 2010; Ran et al., 2014). The mean amplitudes of the P300 were entered into a $3 \times 3 \times 3$ repeated-measures ANOVA with infant expression and hemisphere (right vs. middle vs. left) as the within-participants factors and attachment style as the betweenparticipants factor.

\section{RESULTS}

\section{Behavioral Results}

An ANOVA of the correct RTs revealed a main effect for infant expression $\left[F(2,124)=3.91, p=0.023, \eta^{2}=0.059\right]$, 


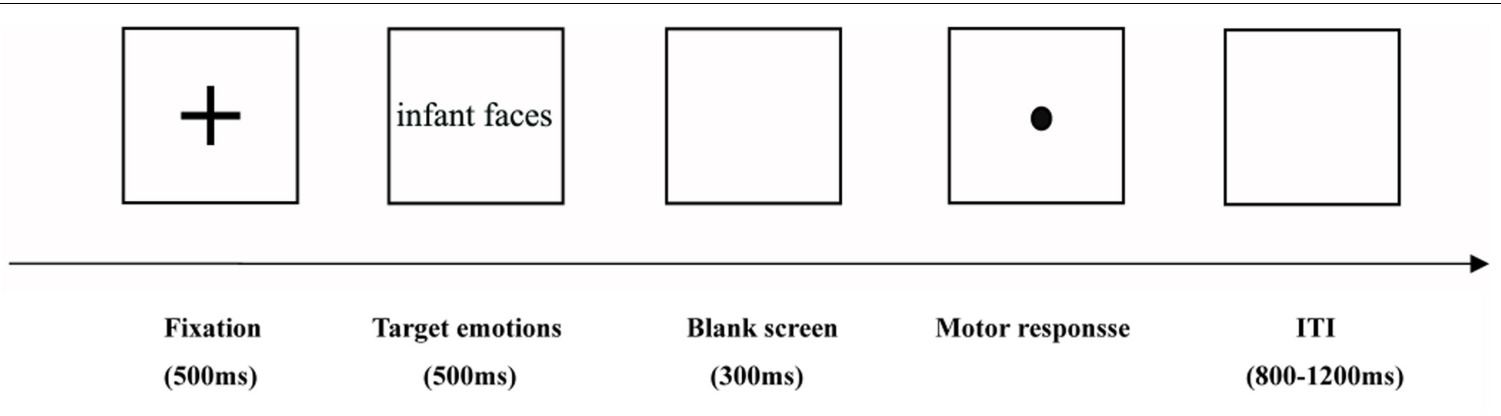

FIGURE 1 | Schematic illustration of the experimental procedure.

indicating that participants responded faster to crying infant faces (293.92 $\pm 13.16 \mathrm{~ms})$ than neutral infant faces $(306.15 \pm 13.24 \mathrm{~ms}, p=0.041)$. Although the main effect of attachment style was not statistically significant $(p>0.05)$, a trend was found in which securely attached individuals $(285.63 \pm 15.64 \mathrm{~ms})$ exhibited the shortest RTs and anxiously attached individuals (319.66 $\pm 16.85 \mathrm{~ms}$ ) had the longest RTs (avoidant: $293.12 \pm 13.34 \mathrm{~ms}$ ). There was a significant interaction between attachment style and infant facial expression $\left[F(4,124)=2.46, p=0.049, \eta^{2}=0.073\right]$, but a simple effects analysis indicated that there were no differences among attachment styles for the infant faces. While the analysis of infant facial expressions within each attachment style showed that securely attached participants' responses to joy $(277.97 \pm 24.45 \mathrm{~ms})$ and crying $(281.05 \pm 23.60 \mathrm{~ms})$ infant faces were faster than to neutral ones $(297.87 \pm 23.74 \mathrm{~ms})(p=0.006$ and 0.056, respectively), anxiously attached participants' responses to crying $(306.23 \pm 23.60 \mathrm{~ms})$ infant faces were faster than to joy $(324.03 \pm 24.45 \mathrm{~ms})$ and neutral ones (328.72 $\pm 23.74 \mathrm{~ms})(p=0.032$ and 0.012 , respectively), while there was no significant difference among infant facial expressions in avoidantly attached participants (joy: $292.99 \pm 21.87 \mathrm{~ms}$; neutral: $291.86 \pm 21.23 \mathrm{~ms}$; crying: $294.47 \pm 21.11 \mathrm{~ms})$. The corresponding ANOVA of the accuracy rates did not yield any significant effects.

\section{ERP Results N170 Effect}

An ANOVA of the N170 latency revealed a significant main effect of infant facial expression $[F(2,124)=17.03, p<0.001$, $\left.\eta^{2}=0.216\right]$, reflecting longer latencies for crying infant faces than for joy $(155.09 \pm 1.20 \mathrm{~ms})$ and neutral $(155.04 \pm 1.18 \mathrm{~ms})$ ones (both $p<0.001$ ). Similarly, the corresponding ANOVA of the N170 peak amplitude revealed a significant main effect of infant facial expression $\left[F(2,124)=5.55, p=0.008, \eta^{2}=0.082\right]$, with statistically significant larger amplitudes for joy infant faces $(-8.23 \pm 0.88 \mu \mathrm{V})$ than for crying ones $(-7.79 \pm 0.85 \mu \mathrm{V})$ $(p<0.001)$ and marginally significant larger amplitudes than for neutral ones $(-7.90 \pm 0.86 \mu \mathrm{V})(p=0.073)$. More importantly, we found a significant interaction between attachment style and infant facial expression $\left[F(4,124)=2.83, p=0.036, \eta^{2}=0.084\right]$. Simple effects analyses indicated that the amplitudes evoked in the anxiously attached group (joy: $-10.81 \pm 1.41 \mu \mathrm{V}$; neutral: $-10.18 \pm 1.37 \mu \mathrm{V}$; crying: $-9.80 \pm 1.37 \mu \mathrm{V}$ ) were significantly larger than those of the avoidantly attached group that were evoked by all infant faces (joy: $-5.72 \pm 1.57 \mu \mathrm{V}$; neutral: $-5.80 \pm 1.54 \mu \mathrm{V}$; crying: $-5.67 \pm 1.53 \mu \mathrm{V})(p=0.019$, 0.038 , and 0.048 , respectively). Additionally, although the amplitudes of the securely attached group for all infant faces (joy: $-8.17 \pm 1.57 \mu \mathrm{V}$; neutral: $-7.72 \pm 1.54 \mu \mathrm{V}$; crying: $-7.89 \pm 1.53 \mu \mathrm{V}$ ) were at the mid-level between the anxiously and securely attached group, no differences were found in the N170 amplitudes in response to infants' faces between the securely and avoidantly or anxiously attached group (Figure 2). Significant effects related to attachment style were not found for the hemisphere and they are therefore not reported here. No other main effects or interaction effects were observed for latency or amplitude differences for this component.

\section{P300 Effect}

Analysis of the P300 mean amplitudes indicated a significant main effect of infant facial expression $[F(2,124)=4.06, p=0.021$, $\left.\eta^{2}=0.062\right]$, indicating that the amplitudes for crying infant faces $(6.89 \pm 0.37 \mu \mathrm{V})$ were larger than those for joy $(6.64 \pm 0.35 \mu \mathrm{V})$ and neutral ones $(6.58 \pm 0.36 \mu \mathrm{V})(p=0.050$ and 0.047, respectively). There was also a main effect of attachment style $\left[F(2,62)=3.66, p=0.031, \eta^{2}=0.106\right]$, with larger amplitudes being elicited in the securely attached group $(7.61 \pm 0.63 \mu \mathrm{V})$ than the avoidantly attached group $(5.34 \pm 0.63 \mu \mathrm{V})(p=0.041)$. There were no significant differences between the anxiously $(7.15 \pm 0.57 \mu \mathrm{V})$ and securely or avoidantly attached group ( $p>0.05$ for both). Furthermore, the interaction between attachment style and infant facial expression was also significant $\left[F(4,124)=3.14, p=0.019, \eta^{2}=0.092\right]$, with amplitudes evoked in the securely attached group (joy: $7.59 \pm 0.63 \mu \mathrm{V}$; neutral: $7.30 \pm 0.65 \mu \mathrm{V}$; crying: $7.95 \pm 0.66 \mu \mathrm{V}$ ) being significantly larger than those of the avoidantly attached group (joy: $5.44 \pm 0.63 \mu \mathrm{V}$; neutral: $5.36 \pm 0.65 \mu \mathrm{V}$; crying: $5.23 \pm 0.66 \mu \mathrm{V})$ for all infant faces $(p=0.018,0.038$, and 0.005 , respectively). The amplitudes of the anxiously attached group $(7.49 \pm 0.59 \mu \mathrm{V})$ were larger than those of the avoidantly attached group for crying faces $(p=0.013)$. Moreover, we found that the amplitudes evoked by a crying infant face were greater than those evoked by neutral ones in the securely attached 


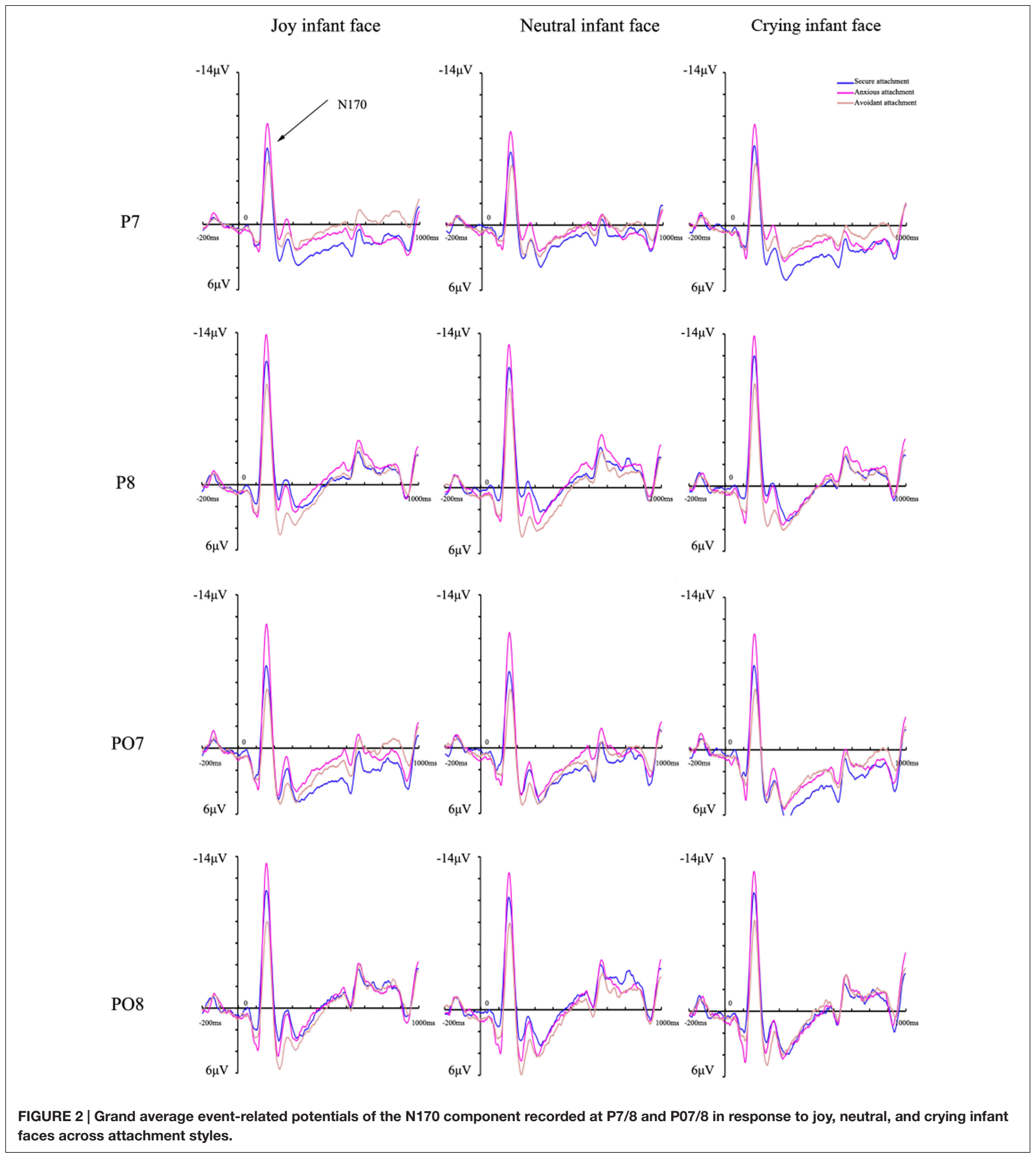

group $(p=0.006)$, and the anxiously attached group also exhibited higher amplitudes for crying than for neutral and joy infant faces $(p<0.05)$. No differences were found for the avoidantly attached group (Figure 3 ). Significant effects related to attachment style were not found for the hemisphere and they are therefore not reported here. No other main effects or interaction effects were observed for amplitude differences for this component. The grand average ERP topographies of the N170 and P300 components in each condition are shown in Figure 4. 


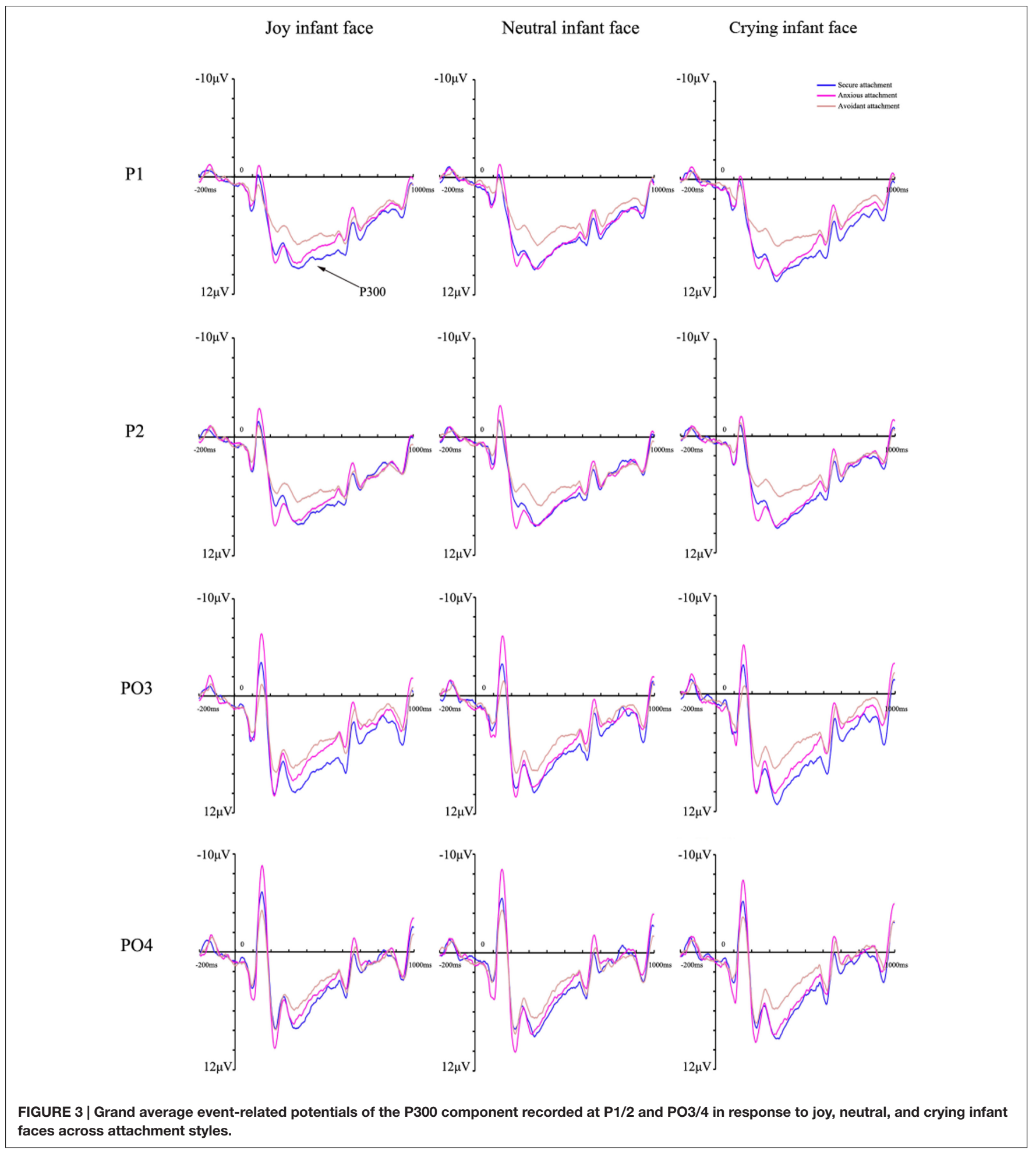

\section{DISCUSSION}

The current study aimed to examine whether the neurophysiological processing of different infant facial expressions by nulliparous females was affected by their attachment styles. On the basis of previous studies, we hypothesized N170 amplitudes of anxiously attached individuals were larger than those of avoidantly attached individuals for all infant emotions; for P300 component, we assumed securely attached individuals would exhibit larger P300 amplitudes than 


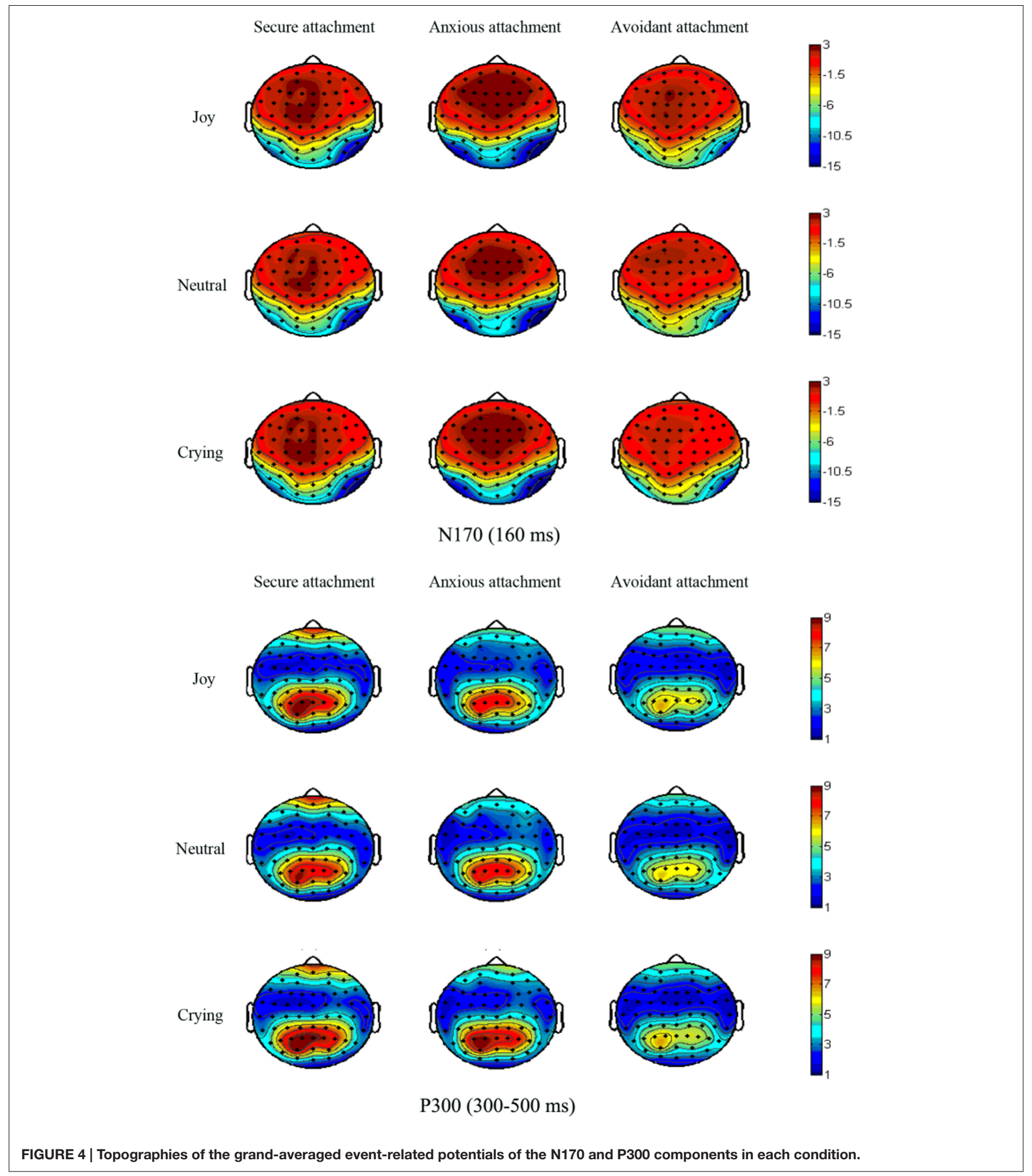

avoidantly attached individuals in all infant emotions, while we expected larger P300 amplitudes in anxiously attached individuals than in avoidantly attached individuals for crying infant faces.
In line with our hypothesis, the ERP results indicated interesting differences between attachment groups. Therefore, specialized neural and cognitive mechanisms that may be involved in the processing of infant faces seem to differ for each 
attachment style. Regarding anxious attachment, a previous study showed that insecurely attached mothers displayed enhanced N170 amplitudes only if the target facial stimuli were imbedded in frequent non-facial stimuli (Fraedrich et al., 2010). In order to exclude the effect of non-facial stimuli, Leyh et al. (2016a) adopted an oddball paradigm with positive, neutral, and negative infant faces, and found that insecurely attached mothers produced larger N170 amplitudes than securely attached mothers during conditions with a negative target stimulus. However, neither study differentiated anxious attachment from avoidant attachment, both of which were conceived as one central dimension of attachment insecurity. The present research examined further this phenomenon and found that the anxiously attached group exhibited more negative N170 amplitudes than the avoidantly attached group in response to all infant faces. The findings of the N170 amplitudes were consistent with the behavioral results, as the RTs to infant faces of the anxiously attached group were the longest among the three attachment styles. Enhanced N170 amplitudes exhibited by the anxiously attached group might represent a greater need for face discrimination resources (Eimer, 2000) since N170 amplitudes increase when faces are attended to Fraedrich et al. (2010).

Additionally, the anxiously attached group exhibited more positive P300 amplitudes than the avoidantly attached group for crying infant faces, which indicates that a processing bias in the anxiously attached group was only found for crying infant faces. However, what puzzled us is that why a processing bias in the anxiously attached group was not found for joy and neutral faces. As mentioned above, anxiously attached individuals preferentially use hyperactive strategies, so they tend to heighten the effect of attachment-related stimuli due to their hypersensitivity (Mikulincer and Shaver, 2007). Consequently, anxiously attached females devote more cognitive resources to the configural processing of infant faces. For example, several studies have indicated that, at an automatic processing level, anxiously attached individuals are more responsive to emotional facial signals than securely attached individuals (Vrtička et al., 2008; Donges et al., 2012). Therefore, we suspect that this great need for face discrimination resources may lead to less motivational engagement and mental resource allocation to joy and neutral infant faces.

Anxiously attached group has greater P300 amplitudes than avoidantly attached group in crying infant faces. This result is easily understood because anxiously attached individuals are characterized by a strong need for closeness, worrying about relationships, and fearing of being rejected (Mikulincer and Shaver, 2007). It is crucial to focus on threat information, such as a crying infant face, in order to maintain relationships. Previous studies have revealed that anxiously attached individuals exhibit larger P300 amplitudes when the oddballs are angry faces in a background of frequently presented neutral stimuli (Mark et al., 2012) and individuals scoring high on attachment anxiety elicit greater Late Positive Potential amplitudes to negative pictures than those scoring low on attachment anxiety (Zilber et al., 2007). Brain imaging research has also revealed that anxiously attached participants exhibit increased activity in the anterior temporal pole, hippocampus, and dorsal anterior cingulate cortex when thinking about negative emotions, while less activity in the orbitofrontal cortex (OFC) when suppressing such thoughts (Gillath et al., 2005). Similarly, anxiously attached participants show increased activation in the anterior insula and dorsal anterior cingulate cortex during social rejection (DeWall et al., 2012) and a left amygdala response is evoked by angry faces when associated with negative feedback (Vrtička et al., 2008). This evidence also converges with the N170 findings which mirrors the correlation between anxious attachment and higher arousal to negative social clues. However, it should be noted that the P300 result for anxious attachment does not mean that anxiously attached females are sensitive to infants' signals. As mentioned above, increased attentional resources were found only for crying infant faces because they may represent a threat to the relationship, which is different to secure attachment and the meeting of infants' needs. Therefore, the processing of crying infant faces in the anxiously attached group may be passive.

The hypothesis that secure attachment would be associated with higher sensitivity to infant faces was supported by our findings, as the securely attached group showed larger P300 amplitudes than the avoidantly attached group for all infant faces. The P300 amplitudes seem to be closely linked to motivational engagement and psychological resource allocation (DuncanJohnson and Donchin, 1977; Polich and Kok, 1995). Accordingly, heightened P300 amplitudes in securely attached females appear to reflect the allocation of considerably more motivated attention to infant faces. Therefore, securely attached females seem to be better able to perceive infant faces which is a crucial component of sensitivity (Fraedrich et al., 2010). Indeed, previous studies have also suggested that more pronounced P300 amplitudes are evoked by infant faces in securely attached mothers in comparison with insecurely attached mothers (Fraedrich et al., 2010; Leyh et al., 2016a). Moreover, neuroimaging evidence has demonstrated that secure attachment is associated with stronger activation, or increased gray matter volume, in the reward network as well as other interconnected regions, such as the hypothalamus or OFC (Vrtička and Vuilleumier, 2012). For example, securely attached mothers exhibited higher ventral striatum and medial OFC activation than avoidantly attached mothers when seeing images of their own babies (Strathearn et al., 2009). Likewise, as for mothers who score higher on the mother's positive perception subscale of the Yale Inventory of Parental Thoughts and Actions, there is not only increased gray matter volume, but also more activity in the OFC in response to infant cries (Kim et al., 2010). Indeed, appropriate perception and interpretation of emotion is considered as an essential component of secure attachment (Riem et al., 2012). Therefore, securely attached individuals pay more attention to the infant's emotional expressions and needs, enabling them to be better at handling their infant's emotions, which at the same time predicts a high level of sensitivity to the infant's signals (Spangler et al., 2010).

Avoidantly attached females were associated with a restricted ability in terms of perceiving infants' faces, as they displayed lower N170 and P300 amplitudes for all infant faces. Indeed, avoidant attachment is linked to a preferential use of deactivating 
strategies to regulate emotions (Mikulincer and Shaver, 2007), allowing the individual to keep the attachment system in a low activation state and prevent others from perceiving their internal emotional states (Vrtička et al., 2012). For instance, Dan and Raz (2012) found significant differences in the C1 and P1 amplitudes in response to angry compared with neutral faces within only avoidantly attached group. The $\mathrm{C} 1$ component (50-100 ms poststimulus) is the first ERP component triggered by the appearance of a stimulus in the visual field and is thought to be pre-attentive and independent of spatial attention (Stolarova et al., 2006). And the $\mathrm{P} 1$ component, an early sensory component peaking around $100 \mathrm{~ms}$ post-stimulus, may constitute an index of mobilization of automatic attentional resources (Hopfinger and Mangun, 2001). Therefore, this study indicated that at early perceptual stage, vigilant rather than defensive style is characteristic of avoidant attachment. Moreover, insecurely attached individuals (including three quarters of avoidantly attached individuals) show a larger P1 and attenuated N170 component over the right hemisphere in response to faces (Escobar et al., 2013). Likewise, the neural findings dovetail nicely with the ERP evidence that avoidantly attached individuals activate motor, mirror, and limbic brain areas to a significantly greater extent, but deactivate the medial OFC and the perigenual anterior cingulate cortex (Lenzi et al., 2013). Cecchini et al. (2015) also reported that avoidantly attached individuals showed a correlation with lower activity of the right temporal and limbic areas. Combining these findings mentioned above, we can infer that avoidantly attached individuals might be characterized by a vigilance-avoidance model. Accordingly, avoidantly attached individuals may adopt an avoiding bias in the processing of infant faces (Vrtička et al., 2008; Lenzi et al., 2013), which fits well with the assumptions and empirical findings regarding avoidant attachment.

To date, relatively rare research has examined how adult attachment styles impact the neurophysiological processing of infant faces by nulliparous females. Given the important individual differences in the functioning of emotional processing among attachment styles, and the fact that existing studies did not differentiate anxious and avoidant attachment from insecure attachment, it is worthy of investigating whether the processing of infant face stimuli is affected by individuals' attachment style, especially in terms of anxious and avoidant attachment. Understanding what role the attachment style plays in the dynamic processing of infant faces can help us to better understand the sensitivity of females with different attachment styles. The current results showed that securely attached females are associated with a high sensitivity to infants' emotions, whereas anxiously and avoidantly attached females are associated with a low sensitivity to infants' signals. As infants rely on the quality of the caregiver's responses to their emotional needs, they may begin

\section{REFERENCES}

Ammaniti, M., Van Ijzendoorn, M. H., Speranza, A. M., and Tambelli, R. (2000). Internal working models of attachment during late childhood and early adolescence: an exploration of stability and change. Attach. Hum. Dev. 2, 328-346. doi: 10.1080/14616730010001587 to evaluate the emotional expressions of their interaction partners in a certain way and learn to express emotions and behavior in the same way (Fraedrich et al., 2010). Therefore, nulliparous females' sensitivity during their interactions with infants is crucial as they will play the role of mother in the future. Moreover, it will be interesting to extend our conclusion to non-nulliparous female, as females recruited in present study are college students whose caregiving system is already mature (Ammaniti et al., 2000; Grossmann et al., 2006; Lenzi et al., 2013), but this extension needs further validation. A limitation of the present study is that the ECR questionnaire does not allow the identification of a specific attachment style because it is a dimensional quantitative scale. In future studies, the researchers need to conduct a clinical assessment using the Adult Attachment Interview to identify a specific attachment style. Furthermore, a clinical assessment of the participants should be conducted because the traits of anxiety and depression may also contribute to the perception of infant facial expressions.

Our findings offer a glimpse into the neurophysiological mechanisms involved in the processing of infant faces according to different attachment styles, and especially further uncover the neurophysiological processing characteristics of anxious and avoidant attachment styles for infant faces. As appropriate perception and interpretation of infants' emotions are considered to be a crucial component of the caregiving system, therefore, different neurophysiological processing of infant faces within the attachment styles may predict females' varying sensitivity to infants' signals (Wolff and Ijzendoorn, 1997).

\section{AUTHOR CONTRIBUTIONS}

YM, XC, GR, and HM performed the data analysis and wrote, reviewed, and approved the manuscript. YM and XC designed the research. NH performed the data collection.

\section{FUNDING}

This project was supported by the National Natural Science Foundation of China (No. 31671149).

\section{ACKNOWLEDGMENTS}

The authors are grateful to Guangming Ran for his help with setting up and running the experiments, to $\mathrm{NH}$ for help during the EEG recordings, and to HM for her help with writing the manuscript. We also thank the participants in the study.

Bakermans-Kranenburg, M. J., Van Ijzendoorn, M. H., and Juffer, F. (2003). Less is more: meta-analyses of sensitivity and attachment interventions in early childhood. Psychol. Bull. 129, 195. doi: 10.1037/0033-2909.129. 2.195

Bowlby, J. (1969). Attachment and Loss: Attachment, Vol. 2. New York, NY: Basic Books. 
Brennan, K. A., Clark, C. L., and Shaver, P. R. (1998). "Self-report measurement of adult attachment: an integrative overview," in Attachment Theory and Close Relationships, eds J. A. Simpson and W. S. Rholes (New York, NY: Guilford Press), 46-76.

Cecchini, M., Iannoni, M. E., Pandolfo, A. L., Aceto, P., and Lai, C. (2015). Attachment style dimensions are associated with brain activity in response to gaze interaction. Soc. Neurosci. 10, 282-293. doi: 10.1080/17470919.2014. 998344

Chavis, J. M., and Kisley, M. A. (2012). Adult attachment and motivated attention to social images: attachment-based differences in event-related brain potentials to emotional images. J. Res. Pers. 46, 55-62. doi: 10.1016/j.jrp.2011.12.004

Cheng, G., Zhang, D. J., Guan, Y. S., and Chen, Y. H. (2015). Preliminary establishment of the standardized Chinese infant facial expression of emotion. Chin. Ment. Health J. 29, 406-412.

Dan, O., and Raz, S. (2012). Adult attachment and emotional processing biases: an Event-Related Potentials (ERPs) study. Biol. Psychol. 91, 212-220. doi: 10.1016/ j.biopsycho.2012.06.003

DeWall, C. N., Masten, C. L., Powell, C., Combs, D., Schurtz, D. R., and Eisenberger, N. I. (2012). Do neural responses to rejection depend on attachment style? An fMRI study. Soc. Cogn. Affect. Neurosci. 7, 184-192. doi: $10.1093 /$ scan/nsq107

Donges, U. S., Kugel, H., Stuhrmann, A., Grotegerd, D., Redlich, R., Lichev, V., et al. (2012). Adult attachment anxiety is associated with enhanced automatic neural response to positive facial expression. Neuroscience 220, 149-157. doi: 10.1016/j.neuroscience.2012.06.036

Duncan-Johnson, C. C., and Donchin, E. (1977). On quantifying surprise: the variation of event-related potentials with subjective probability. Psychophysiology 14, 456-467. doi: 10.1111/j.1469-8986.1977.tb01312.x

Edelstein, R. S., and Gillath, O. (2008). Avoiding interference: adult attachment and emotional processing biases. Pers. Soc. Psychol. Bull. 34, 171-181. doi: $10.1177 / 0146167207310024$

Eimer, M. (2000). The face-specific N170 component reflects late stages in the structural encoding of faces. Neuroreport 11, 2319-2324. doi: 10.1097/ 00001756-200007140-00050

Eimer, M., and Holmes, A. (2007). Event-related brain potential correlates of emotional face processing. Neuropsychologia 45, 15-31. doi: 10.1016/j. neuropsychologia.2006.04.022

Escobar, M. J., Riverarei, A., Decety, J., Huepe, D., Cardona, J. F., Sigman, M., et al. (2013). Attachment patterns trigger differential neural signature of emotional processing in adolescents. PLOS ONE 8:e70247. doi: 10.1371/journal.pone. 0070247

Fraedrich, E. M., Lakatos, K., and Spangler, G. (2010). Brain activity during emotion perception: the role of attachment representation. Attach. Hum. Dev. 12, 231-248. doi: 10.1080/14616731003759724

George, C., and Solomon, J. (1999). “Attachment and caregiving: the caregiving behavioral system," in Handbook of Attachment: Theory, Research, and Clinical Applications, eds J. Cassidy and P. R. Shaver (New York, NY: Guilford Press), 649-670.

Gillath, O., Bunge, S. A., Shaver, P. R., Wendelken, C., and Mikulincer, M. (2005). Attachment-style differences in the ability to suppress negative thoughts: exploring the neural correlates. Neuroimage 28, 835. doi: 10.1016/j.neuroimage. 2005.06.048

Glocker, M. L., Langleben, D. D., Ruparel, K., Loughead, J. W., Gur, R. C., and Sachser, N. (2009). Baby schema in infant faces induces cuteness perception and motivation for caretaking in adults. Ethology 115, 257-263. doi: 10.1111/j.14390310.2008.01603.x

Grossmann, K., Grossmann, K. E., Spangler, G., Suess, G., and Unzner, L. (1985). Maternal sensitivity and newborns' orientation responses as related to quality of attachment in northern Germany. Monogr. Soc. Res. Child Dev 50, $233-256$.

Grossmann, K. E., Grossmann, K., and Waters, E. (eds) (2006). Attachment From(infancy ) to Adulthood: The Major Longitudinal Studies. New York, NY: Guilford Press.

Herrmann, M., Schreppel, T., Jäger, D., Koehler, S., Ehlis, A. C., and Fallgatter, A. J. (2007). The other-race effect for face perception: an event-related potential study. J. Neural Transm. 114, 951-957. doi: 10.1007/s00702-007-0624-9

Hopfinger, J. B., and Mangun, G. R. (2001). "Electrophysiological studies of reflexive attention," in Attraction, Distraction, and Action: Multiple Perspectives on Attentional Capture, eds C. Folk and B. Gibson (Amsterdam: Elsevier), 3-26. doi: 10.1016/S0166-4115(01)80003-0

Kim, P., Leckman, J. F., Mayes, L. C., Feldman, R., Wang, X., and Swain, J. E. (2010). The plasticity of human maternal brain: longitudinal changes in brain anatomy during the early postpartum period. Behav. Neurosci. 124, 695. doi: $10.1037 / \mathrm{a} 0020884$

Lenzi, D., Trentini, C., Pantano, P., Macaluso, E., Lenzi, G. L., and Ammaniti, M. (2013). Attachment models affect brain responses in areas related to emotions and empathy in nulliparous women. Hum. Brain Mapp. 34, 1399-1414. doi: $10.1002 / \mathrm{hbm} .21520$

Lenzi, D., Trentini, C., Tambelli, R., and Pantano, P. (2015). Neural basis of attachment-caregiving systems interaction: insights from neuroimaging studies. Front. Psychol. 6:1241. doi: 10.3389/fpsyg.2015.01241

Leyh, R., Heinisch, C., Behringer, J., Reiner, I., and Spangler, G. (2016a). Maternal attachment representation and neurophysiological processing during the perception of infants' emotional expressions. PLOS ONE 11:e0147294. doi: 10.1371/journal.pone.0147294

Leyh, R., Heinisch, C., Kungl, M. T., and Spangler, G. (2016b). Attachment representation moderates the influence of emotional context on information processing. Front. Hum. Neurosci. 10:278. doi: 10.3389/fnhum.2016.00278

Lopez, F. G., and Gormley, B. (2002). Stability and change in adult attachment style over the first-year college transition: relations to self-confidence, coping, and distress patterns. J. Couns. Psychol. 49, 355. doi: 10.1037/0022-0167.49.3.355

Luby, J. L., Barch, D. M., Belden, A., Gaffrey, M. S., Tillman, R., Babb, C., et al. (2012). Maternal support in early childhood predicts larger hippocampal volumes at school age. Proc. Natl. Acad. Sci. U.S.A. 109, 2854-2859. doi: 10.1073/pnas.1118003109

Luo, Q. L., Wang, H. L., Dzhelyova, M., Huang, P., and Mo, L. (2016). Effect of affective personality information on face processing: evidence from ERPs. Front. Psychol. 7:810. doi: 10.3389/fpsyg.2016.00810

Mark, R. E., Geurdes, F. I. M., and Bekker, M. H. J. (2012). Attachment styles are related to erps elicited to angry faces in an oddball paradigm. J. Behav. Brain Sci. 2, 128-140. doi: 10.4236/jbbs.2012.21015

Mikulincer, M., Gillath, O., and Shaver, P. R. (2002). Activation of the attachment system in adulthood: threat-related primes increase the accessibility of mental representations of attachment figures. J. Pers. Soc. Psychol. 83, 881. doi: 10.1037/ 0022-3514.83.4.881

Mikulincer, M., and Shaver, P. R. (2007). Attachment in Adulthood: Structure, Dynamics, and Change. New York, NY: Guilford Press.

Obegi, J. H., Morrison, T. L., and Shaver, P. R. (2004). Exploring intergenerational transmission of attachment style in young female adults and their mothers. J. Soc. Pers. Relatsh. 21, 625-638. doi: 10.1080/14616730802500826

Polich, J. (2007). Updating P300: an integrative theory of P3a and P3b. Clin. Neurophysiol. 118, 2128-2148. doi: 10.1016/j.clinph.2007.04.019

Polich, J., and Kok, A. (1995). Cognitive and biological determinants of P300: an integrative review. Biol. Psychol. 41, 103-146. doi: 10.1016/0301-0511(95) 05130-9

Ran, G., Zhang, Q., Chen, X., and Pan, Y. (2014). The effects of prediction on the perception for own-race and other-race faces. PLoS ONE 9:e114011. doi: 10.1371/journal.pone.0114011

Riem, M. M., Bakermans-Kranenburg, M. J., van IJzendoorn, M. H., Out, D., and Rombouts, S. A. (2012). Attachment in the brain: adult attachment representations predict amygdala and behavioral responses to infant crying. Attach. Hum. Dev. 14, 533-551. doi: 10.1080/14616734.2012.727252

Righart, R., and De Gelder, B. (2008). Rapid influence of emotional scenes on encoding of facial expressions: an ERP study. Soc. Cogn. Affect. Neurosci. 3, 270-278. doi: 10.1093/scan/nsn021

Schore, A. N. (1994). Affect Regulation and the Origin of the Self. Hillsdale, NJ: Lawrence Erlbaum Associates.

Schupp, H. T., Cuthbert, B. N., Bradley, M. M., Cacioppo, J. T., Ito, T., and Lang, P. J. (2000). Affective picture processing: the late positive potential is modulated by motivational relevance. Psychophysiology 37, 257-261. doi: 10.1111/14698986.3720257

Sonnby-Borgström, M., and Jönsson, P. (2004). Dismissing-avoidant pattern of attachment and mimicry reactions at different levels of information processing. Scand. J. Psychol. 45, 103-113. doi: 10.1111/j.1467-9450.2004.00385.x

Spangler, G., Maier, U., Geserick, B., and von Wahlert, A. (2010). The influence of attachment representation on parental perception and interpretation of infant 
emotions: a multilevel approach. Dev. Psychobiol. 52, 411. doi: 10.1002/dev. 20441

Stolarova, M., Keil, A., and Moratti, S. (2006). Modulation of the C1 visual event-related component by conditioned stimuli: evidence for sensory plasticity in early affective perception. Cereb. Cortex 16, 876-887. doi: 10.1093/cercor/ bhj031

Strathearn, L., Fonagy, P., Amico, J., and Montague, P. R. (2009). Adult attachment predicts maternal brain and oxytocin response to infant cues. Neuropsychopharmacology 34, 2655-2666. doi: 10.1038/npp.2009.103

Swain, J. E. (2008). Baby stimuli and the parent brain: functional neuroimaging of the neural substrates of parent-infant attachment. Psychiatry 5, 28-36.

Swain, J. E., Kim, P., and Ho, S. S. (2011). Neuroendocrinology of parental response to baby-cry. J. Neuroendocrinol. 23, 1036-1041. doi: 10.1111/j.1365-2826.2011. 02212.x

Swain, J. E., Kim, P., Spicer, J., Ho, S. S., Dayton, C. J., Elmadih, A., et al. (2014). Approaching the biology of human parental attachment: brain imaging, oxytocin and coordinated assessments of mothers and fathers. Brain Res. 1580, 78-101. doi: 10.1016/j.brainres.2014.03.007

Tacikowski, P., and Nowicka, A. (2010). Allocation of attention to self-name and self-face: an ERP study. Biol. Psychol. 84, 318-324. doi: 10.1016/j.biopsycho. 2010.03.009

van Ijzendoorn, M. (1995). Adult attachment representations, parental responsiveness, and infant attachment: a meta-analysis on the predictive validity of the Adult Attachment Interview. Psychol. Bull. 117, 387. doi: 10.1037/0033-2909.117.3.387

Vrtička, P., Andersson, F., Grandjean, D., Sander, D., and Vuilleumier, P. (2008). Individual attachment style modulates human amygdala and striatum activation during social appraisal. PLOS ONE 3:e2868. doi: 10.1371/journal. pone. 0002868

Vrtička, P., Bondolfi, G., Sander, D., and Vuilleumier, P. (2012). The neural substrates of social emotion perception and regulation are modulated by adult attachment style. Soc. Neurosci. 7, 473-493. doi: 10.1080/17470919.2011. 647410

Vrtička, P., and Vuilleumier, P. (2012). Neuroscience of human social interactions and adult attachment style. Front. Hum. Neurosci. 6:212. doi: 10.3389/fnhum. 2012.00212

Whittle, S., Vijayakumar, N., Dennison, M., Schwartz, O., Simmons, J. G., Sheeber, L., et al. (2015). "Effects of positive and negative parenting behaviors on brain development from early to late adolescence: a longitudinal structural brain imaging study," in Biological Psychiatry, Vol. 77, eds H. John and M. D. Krystal (New York, NY: Elsevier Science Inc).

Wolff, M. S., and Ijzendoorn, M. H. (1997). Sensitivity and attachment: a metaanalysis on parental antecedents of infant attachment. Child Dev. 68, 571-591. doi: 10.1111/j.1467-8624.1997.tb04218.x

Zhang, X., Li, T., and Zhou, X. (2008). Brain responses to facial expressions by adults with different attachment-orientations. Neuroreport 19, 437-441. doi: 10.1097/WNR.0b013e3282f55728

Zilber, A., Goldstein, A., and Mikulincer, M. (2007). Adult attachment orientations and the processing of emotional pictures-erp correlates. Pers. Individ. Dif. 43, 1898-1907. doi: 10.1016/j.paid.2007.06.015

Conflict of Interest Statement: The authors declare that the research was conducted in the absence of any commercial or financial relationships that could be construed as a potential conflict of interest.

Copyright (C) 2017 Ma, Ran, Chen, Ma and Hu. This is an open-access article distributed under the terms of the Creative Commons Attribution License (CC BY). The use, distribution or reproduction in other forums is permitted, provided the original author(s) or licensor are credited and that the original publication in this journal is cited, in accordance with accepted academic practice. No use, distribution or reproduction is permitted which does not comply with these terms. 